\title{
A NONTRIVIAL ALGEBRAIC CYCLE IN THE JACOBIAN VARIETY OF THE FERMAT SEXTIC
}

\author{
By \\ Yuuki TADOKORO
}

\begin{abstract}
We compute some value of the harmonic volume for the Fermat sextic. Using this computation, we prove that some special algebraic cycle in the Jacobian variety of the Fermat sextic is not algebraically equivalent to zero.
\end{abstract}

\section{Introduction}

B. Harris [5] defined the harmonic volume for the compact Riemann surface $X$ of genus $g \geq 3$, using Chen's iterated integrals [2]. Let $J(X)$ be the Jacobian variety of $X$. By the Abel-Jacobi map $X \rightarrow J(X), X$ is embedded in $J(X)$. By a consideration of the special harmonic volume, Harris [6] proved that the algebraic cycle $F(4)-F(4)^{-}$is not algebraically equivalent to zero in $J(F(4))$. Here, $F(4)$ is the Fermat quartic, which is a compact Riemann surface of genus 3. Ceresa [1] showed that the algebraic cycle $X-X^{-}$is not algebraically equivalent to zero in $J(X)$ for a generic $X$. We know few explicit nontrivial examples except for $F(4)$. Harris [7] used the special feature of $F(4)$ that its normalized period matrix has entries in a discrete subring of $\mathbf{C}$. The Fermat sextic $F(6)$ has the same feature. We use this and prove

THeOREM 4.3. Let $F(6)$ be the Fermat sextic. Then, the algebraic cycle $F(6)-F(6)^{-}$is not algebraically equivalent to zero in $J(F(6))$.

We compute iterated integrals with some common base point of $F(6)$. This is a similar computation of Tretkoff and Tretkoff [10]. In order to compute the Poincaré dual of $F(6)$, we use the result of Kamata [8] for the intersection number of the first integral homology class of the Fermat curves. It is difficult to

Received October 30, 2007

Revised April 3, 2009. 
apply Harris' method to other Fermat curves. We guess that we need to extend the harmonic volume, but it has not been extended so far. We [9] proved the same fact as the Klein quartic, but we did not use the above special feature.

Now we describe the contents of this paper briefly. In $\S 2$, we recall the definition and fundamental properties of the harmonic volume and algebraic cycle in $J(X) . \S 3$ is devoted to the computation of iterated integrals of the Fermat curves. In the latter half of this section, we prove that iterated integrals on those curves are represented by some special values of the generalized hypergeometric function ${ }_{3} F_{2}$. It was introduced in [9] but not proved. In $\S 4$, we prove Main Theorem, using the numerical calculation by the Mathematica program.

\section{Acknowledgments}

The author is grateful to Nariya Kawazumi for valuable advice and support. This work is partially supported by 21st Century COE program (University of Tokyo) by the Ministry of Education, Culture, Sports, Science and Technology.

\section{Harmonic Volumes and Algebraic Cycles}

Let $R$ be a discrete subring of $\mathbf{C}$. We suppose that all the entries of the period matrix of the compact Riemann surface $X$ can be reduced to elements of $R$. Harris [7] pointed out that we may replace $\mathbf{Z}[\sqrt{-1}]$ for the Fermat quartic in Harris' method in [6] with $R$. We recall the harmonic volume for such $X$ as follows. Let $H_{R}^{1,0}$ denote the space of homolophic 1-forms on $X$ with $R$-periods. It is a $g$-dimensional $\mathbf{C}$-vector space. We choose a basis $\left\{K_{1}, K_{2}, \ldots, K_{2 g}\right\}$ of the first integral homology group $H_{1}(X ; \mathbf{Z})$ of $X$.

Definition 2.1 ([7]). The harmonic volume is defined to be the homomorphism $\left(H_{R}^{1,0}\right)^{\otimes_{R} 3} \rightarrow \mathbf{C} / R$ by

$$
I_{R}\left(\omega_{1} \otimes \omega_{2} \otimes \omega_{3}\right)=\sum_{r=1}^{2 g} a_{r} \int_{C_{r}} \omega_{1} \omega_{2} \quad \bmod R .
$$

Here $\omega_{1} \otimes \omega_{2} \otimes \omega_{3}$ is an element of $\left(H_{R}^{1,0}\right)^{\otimes_{R} 3}, C_{r}$ is a loop in $X$ at the fixed base point $x_{0}$ whose homology class is $K_{r}$, and the Poincaré dual of $\omega_{3}$ is equal to $\sum_{r=1}^{2 g} a_{r} K_{r}\left(a_{r} \in \mathbf{C}\right)$. The integral $\int_{C_{r}} \omega_{1} \omega_{2}$ is Chen's iterated integral [2], that is, $\int_{C_{r}} \omega_{1} \omega_{2}=\int_{0 \leq t_{1} \leq t_{2} \leq 1} f_{1}\left(t_{1}\right) f_{2}\left(t_{2}\right) d t_{1} d t_{2}$ for $C_{r}^{*} \omega_{i}=f_{i}(t) d t, i=1,2$, where $t$ is the coordinate in the unit interval $[0,1]$. 
A nontrivial algebraic cycle in the Jacobian variety of the Fermat sextic 31

We remark that $I_{R}$ dose not depend on the choice of the base point $x_{0}$. It is a modified version of the original harmonic volume $I$. See Harris [5] for $I$.

Let $J=J(X)$ be the Jacobian variety of $X$. By the Abel-Jacobi map $X \rightarrow J(X), X$ is embedded in $J(X)$. The algebraic 1-cycle $X-X^{-}$in $J(X)$ is homologous to zero. Here we denote by $X^{-}$the image of $X$ under the multiplication map by -1 . We recall the relation between the harmonic volume and algebraic 1-cycle $X-X^{-}$in $J$. We say the algebraic cycle $X-X^{-}$is algebraically equivalent to zero in $J$ if there exists a topological 3-chain $W$ such that $\partial W=X-X^{-}$and $W$ lies on $S$, where $S$ is an algebraic (or complex analytic) subset of $J$ of complex dimension 2 (Harris [7]). The chain $W$ is unique up to 3-cycles. Harris proved the key theorem.

TheOREM 2.2 (Section 2.7 in [7]). If the algebraic cycle $X-X^{-}$is algebraically equivalent to zero in $J$, then $2 I_{R}(\omega) \equiv 0$ modulo $R$ for any $\omega \in\left(H_{R}^{1,0}\right)^{\otimes_{R} 3}$.

See Harris [6, 7] for details. In $\S 4$, we find some element $\omega \in\left(H_{R}^{1,0}\right)^{\otimes_{R} 3}$ such that $2 I_{R}(\omega) \not \equiv 0$ modulo $R$ for the Fermat sextic.

\section{Iterated Integrals of the Fermat Curves}

In this section we compute iterated integrals of the Fermat curves. Let $H^{1,0}$ denote the space of holomorphic 1 -forms on $X$. We choose a basis $\left\{\omega_{1}, \omega_{2}, \ldots\right.$, $\omega_{g}$ \} of $H^{1,0}$. Let $\gamma$ be a loop in $X$ at some base point. We remark that the iterated integral $\int_{\gamma} \omega_{i} \omega_{j}$ depends on the choice of the base points and is invariant under homotopy relative a fixed base point. This iterated integral and the quadratic period defined by Gunning [4] are essentially same except for the sign.

For $N \in \mathbf{Z}_{\geq 3}$, let $F(N)=\left\{(X: Y: Z) \in \mathbf{C} P^{2} ; X^{N}+Y^{N}=Z^{N}\right\}$ denote the Fermat curve of degree $N$, which is a compact Riemann surface of genus $(N-1)(N-2) / 2$. Let $x$ and $y$ denote $X / Z$ and $Y / Z$ respectively. The equation $X^{N}+Y^{N}=Z^{N}$ induces $x^{N}+y^{N}=1$. Using this coordinate $(x, y) \in F(N)$, the holomorphic map $\pi: F(N) \rightarrow \mathbf{C} P^{1}$ is defined by $\pi(x, y)=x$. It is clear that $\pi$ is an $N$-sheeted covering $F(N) \rightarrow \mathbf{C} P^{1}$, branched over $N$ branch points $\left\{\zeta_{N}^{i}\right\}_{i=0,1, \ldots, N-1} \subset \mathbf{C} P^{1}$. Here $\zeta_{N}$ denotes $\exp (2 \pi \sqrt{-1} / N)$. Holomorphic automorphisms $\alpha$ and $\beta$ of $F(N)$ are defined by $\alpha(X: Y: Z)=\left(\zeta_{N} X: Y: Z\right)$ and $\beta(X: Y: Z)=\left(X: \zeta_{N} Y: Z\right)$ respectively. We have that $\alpha \beta=\beta \alpha$ and the subgroup of the holomorphic automorphisms of $F(N)$ which is generated by $\alpha$ and $\beta$ is isomorphic to $(\mathbf{Z} / N \mathbf{Z}) \times(\mathbf{Z} / N \mathbf{Z})$. Let $P_{i}$ and $Q_{i}$ denote $\alpha^{i}(1,0)$ and $\beta^{i}(0,1)$, 
$i=0,1, \ldots, N-1$ respectively. We define a simply connected domain $\Omega$ by $\mathbf{C} \backslash \bigcup_{j=0}^{j=N-1}\left\{t \zeta^{j} ; t \geq 1, t \in \mathbf{R}\right\}$. Then $\pi^{-1}(\Omega)$ consists of $N$ path-connected components and we denote by $\Omega_{i}$ a connected component of $\pi^{-1}(\Omega)$ which contains $Q_{i}, i=0,1, \ldots, N-1$. Let $\gamma_{0}$ be a path $[0,1] \ni t \mapsto\left(t, \sqrt[N]{1-t^{N}}\right) \in F(N)$, where $\sqrt[N]{1-t^{N}}$ is a real nonnegative analytic function on $[0,1]$. A loop in $F(N)$ is defined by

$$
\kappa_{0}=\gamma_{0} \cdot\left(\beta \gamma_{0}\right)^{-1} \cdot\left(\alpha \beta \gamma_{0}\right) \cdot\left(\alpha \gamma_{0}\right)^{-1},
$$

where the product $\ell_{1} \cdot \ell_{2}$ indicates that we traverse $\ell_{1}$ first, then $\ell_{2}$. We consider a loop $\alpha^{i} \beta^{j} \kappa_{0}$ as an element of the first homology group $H_{1}(F(N) ; \mathbf{Z})$ of $F(N)$. Kamata obtained the following lemma for the intersection number of $H_{1}(F(N) ; \mathbf{Z})$.

Lemma 3.1 (Section 5 in [8]). We have

$$
\left\{\begin{array}{l}
\left(\kappa_{0}, \alpha \kappa_{0}\right)=1=-\left(\alpha \kappa_{0}, \kappa_{0}\right) \\
\left(\kappa_{0}, \beta \kappa_{0}\right)=1=-\left(\beta \kappa_{0}, \kappa_{0}\right) \\
\left(\kappa_{0}, \alpha \beta \kappa_{0}\right)=-1=-\left(\alpha \beta \kappa_{0}, \kappa_{0}\right) \\
\left(\kappa_{0}, \alpha \beta^{-1} \kappa_{0}\right)=0=\left(\alpha \beta^{-1} \kappa_{0}, \kappa_{0}\right) .
\end{array}\right.
$$

From this lemma, it is to show

Proposition 3.2 (Section 5 in [8]). We have $\left\{\alpha^{i} \beta^{j} \kappa_{0}\right\}_{i=0,1, \ldots, N-3, j=0,1, \ldots, N-2}$ is a basis of $H_{1}(F(N) ; \mathbf{Z})$.

REMARK 3.3. Intersection matrix of $\left\{\alpha^{i} \beta^{j} \kappa_{0}\right\}_{i=0,1, \ldots, N-3, j=0,1, \ldots, N-2}$ is given by $K$ in case (i) in [8].

It is a known fact that $\left\{\omega_{r, s}^{\prime}=x^{r-1} y^{s-1} d x / y^{N-1}\right\}_{r, s \geq 1, r+s \leq N-1}$ is a basis of $H^{1,0}$ of $F(N)$. The beta function $B(u, v)$ is defined by $\int_{0}^{1} t^{u-1}(1-t)^{v-1} d t$ for $u, v>0$. It is clear that

$$
\int_{\alpha^{i} \beta^{j} \gamma_{0}} \omega_{r, s}^{\prime}=\zeta_{N}^{i r+j s} \int_{\gamma_{0}} \omega_{r, s}^{\prime}=\zeta_{N}^{i r+j s} \frac{B(r / N, s / N)}{N} .
$$

The integral of $\omega_{r, s}^{\prime}$ along $\alpha^{i} \beta^{j} \kappa_{0}$ is obtained as follows.

Proposition 3.4 (Appendix in [3]). We have

$$
\int_{\alpha^{i} \beta^{j} \kappa_{0}} \omega_{r, s}^{\prime}=B(r / N, s / N)\left(1-\zeta_{N}^{r}\right)\left(1-\zeta_{N}^{s}\right) \zeta_{N}^{i r+j s} / N .
$$


A nontrivial algebraic cycle in the Jacobian variety of the Fermat sextic

33

We denote the 1-form $N \omega_{r, s}^{\prime} / B_{r, s}^{N}$ by $\omega_{r, s}$. Here, $B_{r, s}^{N}=B(r / N, s / N)$. This implies $\int_{\alpha^{i} \beta^{j}{ }^{j}{ }_{0}} \omega_{r, s} \in \mathbf{Z}\left[\zeta_{N}\right]$.

Let $f_{r, s}$ be a real 1 -form on $[0,1]$ defined by $\gamma_{0}^{*} \omega_{r, s}^{\prime}=t^{r-1}\left(\sqrt[N]{1-t^{N}}\right)^{s-N} d t$ for $r, s \geq 1, r+s \leq N-1$. The iterated integral $\int_{\gamma_{0}} \omega_{r, s} \omega_{l, m}=N^{2} \int_{\gamma} f_{r, s} f_{l, m} /\left(B_{r, s}^{N} B_{l, m}^{N}\right)$ is denoted by $x_{r, s, l, m}$. Here, $\gamma$ is the path $[0,1] \ni t \mapsto t \in[0,1]$. Iterated integrals of $\omega_{r, s}$ along the loop $\alpha^{i} \beta^{j} \kappa_{0}$ can be computed.

Lemma 3.5. We consider $\alpha^{i} \beta^{j} \kappa_{0}$ as a loop at the base point $Q_{j}$. Then the iterated integral $\int_{\alpha^{i} \beta^{j} \kappa_{0}} \omega_{r, s} \omega_{l, m}$ is given by

$$
\zeta_{N}^{i(r+l)+j(s+m)}\left\{\left(1-\zeta_{N}^{r+l}\right)\left(1-\zeta_{N}^{s+m}\right) x_{r, s, l, m}+\left(1-\zeta_{N}^{s}\right)\left(\zeta_{N}^{r+l}+\zeta_{N}^{l+m}-\zeta_{N}^{m}-\zeta_{N}^{l}\right)\right\} .
$$

PRoOF. It is clear that $\int_{\alpha^{i} \beta^{j} k_{0}} \omega_{r, s} \omega_{l, m}=\zeta_{N}^{i(r+l)+j(s+m)} \int_{\kappa_{0}} \omega_{r, s} \omega_{l, m}$. We have only to compute $\int_{\kappa_{0}} \omega_{r, s} \omega_{l, m}$. We denote $\left(\int_{\ell_{1}}+\int_{\ell_{2}}\right) \omega_{r, s} \omega_{l, m}=\int_{\ell_{1}} \omega_{r, s} \omega_{l, m}+$ $\int_{\ell_{2}} \omega_{r, s} \omega_{l, m}$ only here.

Proposition 3.4 , the equation $\int_{\gamma_{0}} \omega_{r, s}=1$, and

$$
\int_{\gamma_{0}} \omega_{r, s} \omega_{l, m}+\int_{\gamma_{0}^{-1}} \omega_{r, s} \omega_{l, m}+\int_{\gamma_{0}} \omega_{r, s} \int_{\gamma_{0}^{-1}} \omega_{l, m}=\int_{\gamma_{0} \cdot \gamma_{0}^{-1}} \omega_{r, s} \omega_{l, m}=0
$$

give us the equation

$$
\begin{aligned}
\int_{\kappa_{0}} \omega_{r, s} \omega_{l, m}= & \left(\int_{\gamma_{0}}+\int_{\left(\beta \gamma_{0}\right)^{-1}}+\int_{\alpha \beta \gamma_{0}}+\int_{\left(\alpha \gamma_{0}\right)^{-1}}\right) \omega_{r, s} \omega_{l, m} \\
& +\int_{\gamma_{0}} \omega_{r, s}\left(\int_{\left(\beta \gamma_{0}\right)^{-1}}+\int_{\alpha \beta \gamma_{0}}+\int_{\left(\alpha \gamma_{0}\right)^{-1}}\right) \omega_{l, m} \\
& +\int_{\left(\beta \gamma_{0}\right)^{-1}} \omega_{r, s}\left(\int_{\alpha \beta \gamma_{0}}+\int_{\left(\alpha \gamma_{0}\right)^{-1}}\right) \omega_{l, m}+\int_{\alpha \beta \gamma_{0}} \omega_{r, s} \int_{\left(\alpha \gamma_{0}\right)^{-1}} \omega_{l, m} \\
= & \left.\int_{\gamma_{0}}+\zeta_{N}^{s+m} \int_{\gamma_{0}^{-1}}+\zeta_{N}^{r+s+l+m} \int_{\gamma_{0}}+\zeta_{N}^{r+l} \int_{\gamma_{0}^{-1}}\right) \omega_{r, s} \omega_{l, m} \\
& +\int_{\gamma_{0}} \omega_{r, s}\left(-\zeta_{N}^{m} \int_{\gamma_{0}}+\zeta_{N}^{l+m} \int_{\gamma_{0}}-\zeta_{N}^{l} \int_{\gamma_{0}}\right) \omega_{l, m} \\
& -\zeta_{N}^{s} \int_{\gamma_{0}} \omega_{r, s}\left(\zeta_{N}^{l+m} \int_{\gamma_{0}}-\zeta_{N}^{l} \int_{\gamma_{0}}\right) \omega_{l, m}-\left(\zeta_{N}^{r+s} \int_{\gamma_{0}} \omega_{r, s}\right) \zeta_{N}^{l} \int_{\gamma_{0}} \omega_{l, m}
\end{aligned}
$$




$$
\begin{aligned}
= & \left\{\left(1+\zeta_{N}^{r+s+l+m}\right) \int_{\gamma_{0}}-\left(\zeta_{N}^{s+m}+\zeta_{N}^{r+l}\right) \int_{\gamma_{0}}\right\} \omega_{r, s} \omega_{l, m} \\
& +\left(\zeta_{N}^{s+m}+\zeta_{N}^{r+l}\right) \int_{\gamma_{0}} \omega_{r, s} \int_{\gamma_{0}} \omega_{l, m} \\
& -\zeta_{N}^{m}+\zeta_{N}^{l+m}-\zeta_{N}^{l}-\zeta_{N}^{s+l+m}+\zeta_{N}^{s+l}-\zeta_{N}^{r+s+l} \\
= & \left(1-\zeta_{N}^{r+l}\right)\left(1-\zeta_{N}^{s+m}\right) \int_{\gamma_{0}} \omega_{r, s} \omega_{l, m} \\
& +\left(1-\zeta_{N}^{s}\right)\left(\zeta_{N}^{r+l}+\zeta_{N}^{l+m}-\zeta_{N}^{m}-\zeta_{N}^{l}\right) .
\end{aligned}
$$

We define a path $\gamma_{j}$ by $\gamma_{0} \cdot\left(\beta^{j} \gamma_{0}\right)^{-1}$. Let $\gamma_{i, j}$ denote the loop $\gamma_{j} \cdot\left(\alpha^{i} \beta^{j} \kappa_{0}\right) \cdot \gamma_{j}^{-1}$. Using the above lemma, we have iterated integrals of $\omega_{r, s}$ along the loop $\gamma_{i, j}$ at the common base point $Q_{0}$.

THEOREM 3.6. The iterated integral $\int_{\gamma_{i, j}} \omega_{r, s} \omega_{l, m}$ is given by

$$
\begin{gathered}
\zeta_{N}^{i(r+l)+j(s+m)}\left\{\left(1-\zeta_{N}^{l+r}\right)\left(1-\zeta_{N}^{m+s}\right) x_{r, s, l, m}+\left(1-\zeta_{N}^{s}\right)\left(\zeta_{N}^{l+r}+\zeta_{N}^{l+m}-\zeta_{N}^{m}-\zeta_{N}^{l}\right)\right\} \\
+\left(1-\zeta_{N}^{j s}\right)\left(1-\zeta_{N}^{l}\right)\left(1-\zeta_{N}^{m}\right) \zeta_{N}^{i l+j m}-\left(1-\zeta_{N}^{j m}\right)\left(1-\zeta_{N}^{r}\right)\left(1-\zeta_{N}^{s}\right) \zeta_{N}^{i r+j s}
\end{gathered}
$$

Tretkoff and Tretkoff [10] computed the quadratic periods with another base point by similar computation.

Proof. We have

$$
\int_{\gamma_{i, j}} \omega_{r, s} \omega_{l, m}=\int_{\alpha^{i} \beta^{j} \kappa_{0}} \omega_{r, s} \omega_{l, m}+\int_{\gamma_{j}} \omega_{r, s} \int_{\alpha^{i} \beta^{j} \kappa_{0}} \omega_{l, m}-\int_{\alpha^{i} \beta^{j} \kappa_{0}} \omega_{r, s} \int_{\gamma_{j}} \omega_{l, m} .
$$

From this equation and Lemma 3.5, the result follows.

For the numerical calculation of $x_{r, s, l, m}$, we recall the generalized hypergeometric function ${ }_{3} F_{2}$. Let $\Gamma(\tau)$ denote the gamma function $\int_{0}^{\infty} e^{-t} t^{\tau-1} d t$ for $\tau>0$. We define $(\alpha, n)$ by $\Gamma(\alpha+n) / \Gamma(\alpha)$ for $n \in \mathbf{Z}_{\geq 0}$. For $x \in\{z \in \mathbf{C} ;|z|<1\}$ and $\alpha_{1}, \alpha_{2}, \alpha_{3}, \beta_{1}, \beta_{2}>-1$, the generalized hypergeometric function ${ }_{3} F_{2}$ is defined by

$$
{ }_{3} F_{2}\left(\begin{array}{c}
\alpha_{1}, \alpha_{2}, \alpha_{3} \\
\beta_{1}, \beta_{2}
\end{array} ; x\right)=\sum_{n=0}^{\infty} \frac{\left(\alpha_{1}, n\right)\left(\alpha_{2}, n\right)\left(\alpha_{3}, n\right)}{\left(\beta_{1}, n\right)\left(\beta_{2}, n\right)(1, n)} x^{n} .
$$


A nontrivial algebraic cycle in the Jacobian variety of the Fermat sextic 35

Proposition 3.7. Let $\Delta$ be a 1-simplex $\left\{(u, v) \in \mathbf{R}^{2} ; 0 \leq v \leq 1,0 \leq u \leq v\right\}$. If $a, b, p, q>0, b<1$, then we have

$$
\begin{aligned}
& \int_{\Delta} u^{a-1}(1-u)^{b-1} v^{p-1}(1-v)^{q-1} d u d v \\
& \quad=\frac{B(a+p, q)}{a} \lim _{\substack{t \rightarrow 1-0 \\
t \in \mathbf{R}}} 3 F_{2}\left(\begin{array}{c}
a, 1-b, a+p \\
1+a, a+p+q
\end{array} ; t\right) .
\end{aligned}
$$

Proof. Using the equation

$$
\begin{aligned}
\int_{0}^{v} u^{a-1}(1-u)^{b-1} d u & =\int_{0}^{v} \sum_{n=0}^{\infty} u^{a-1}\left(\begin{array}{c}
b-1 \\
n
\end{array}\right)(-u)^{n} d u \\
& =\sum_{n=0}^{\infty} \frac{(1-b, n)}{(1, n)} \int_{0}^{v} u^{n+a-1} d u,
\end{aligned}
$$

we compute as follows:

$$
\begin{aligned}
\int_{0}^{1} v^{p-1} & (1-v)^{q-1} \int_{0}^{v} u^{a-1}(1-u)^{b-1} d u d v \\
& =\int_{0}^{1} v^{p-1}(1-v)^{q-1} \sum_{n=0}^{\infty} \frac{(1-b, n)}{(1, n)} \int_{0}^{v} u^{n+a-1} d u \\
& =\sum_{n=0}^{\infty} \int_{0}^{1} v^{a+p+n-1}(1-v)^{q-1} \frac{(1-b, n)}{(1, n)} \frac{1}{a+n} d v \\
= & \sum_{n=0}^{\infty} B(a+p+n, q) \frac{(1-b, n)}{(1, n)} \frac{1}{a+n} \\
= & \sum_{n=0}^{\infty} \frac{\Gamma(a+p+n) \Gamma(q)}{\Gamma(a+p+q+n)} \frac{(1-b, n)}{(1, n)} \frac{1}{a+n} \\
= & \frac{\Gamma(a+p) \Gamma(q)}{a \Gamma(a+p+q)} \sum_{n=0}^{\infty} \frac{a}{a+n} \frac{\Gamma(a+p+q)}{\Gamma(a+p+q+n)} \frac{\Gamma(a+p+n)}{\Gamma(a+p)} \frac{(1-b, n)}{(1, n)} \\
= & \frac{B(a+p, q)}{a} \lim _{t \rightarrow 1-0}{ }_{3} F_{2}\left(\begin{array}{c}
a, 1-b, a+p \\
1+a, a+p+q
\end{array} ;\right) .
\end{aligned}
$$

From this proposition, we have 
LEMMA 3.8 .

$$
x_{r, s, l, m}=\frac{N^{2} \int_{\gamma} f_{r, s} f_{l, m}}{B_{r, s}^{N} B_{l, m}^{N}}=\frac{N B_{r+l, m}^{N}}{r B_{r, s}^{N} B_{l, m}^{N}} \lim _{\substack{t \rightarrow 1-0 \\
t \in \mathbf{R}}} F_{2}\left(\begin{array}{c}
r / N, 1-s / N,(r+l) / N \\
1+r / N,(r+l+m) / N
\end{array} ; t\right) .
$$

\section{A Nontrivial Algebraic Cycle in $J(F(6))$}

In this section, we consider only the case $N=6$. We compute some value of the harmonic volume for the Fermat sextic $F(6)$. This tells the nontriviality of the algebraic cycle $F(6)-F(6)^{-}$in $J(F(6))$. We have the genus of $F(6)$ is equal to 10 and $\left\{\omega_{r, s}\right\}_{r, s \geq 1, r+s \leq 5}$ is a basis of $H^{1,0}$ of $F(6)$. For the rest of this paper, we denote $\zeta=\zeta_{6}$ and $R=\mathbf{Z}[\zeta]$. Proposition 3.2 gives that a set of loops $\left\{\gamma_{0,0}, \gamma_{0,1}, \ldots, \gamma_{0,4}, \gamma_{1,0}, \gamma_{1,1}, \ldots, \gamma_{1,4}, \gamma_{2,0} \ldots, \gamma_{3,0}, \gamma_{3,1}, \ldots, \gamma_{3,4}\right\}$ may be considered as a basis of the integral homology group $H_{1}(F(6) ; \mathbf{Z})$ of $F(6)$. Let P.D. : $H^{1}(F(6) ; \mathbf{C}) \rightarrow H_{1}(F(6) ; \mathbf{C})$ be the Poincaré dual.

Lemma 4.1. Let $L_{i, k}$ be a linear combination $\sum_{n=0}^{5} \zeta^{n k} \gamma_{i, n}$ in $H_{1}(F(6) ; \mathbf{C})$. Then we have

P.D. $\left(\omega_{1,1}\right)=\frac{1}{122}\left\{(60-13 \zeta) L_{0,1}-(15-49 \zeta) L_{1,1}-(43-51 \zeta) L_{2,1}-(50-21 \zeta) L_{3,1}\right\}$.

Proof. Since $\beta_{*}\left(\gamma_{i, j}\right)=\gamma_{i, j+1}$ as a homology class, we obtain

We have

$$
\beta_{*} L_{i, k}=\zeta^{-k} L_{i, k} \text {. }
$$

$$
\beta_{*}\left(\text { P.D. }\left(\omega_{1,1}\right)\right)=\text { P.D. }\left(\left(\beta^{-1}\right)^{*} \omega_{1,1}\right)=\zeta^{5} \text { P.D. }\left(\omega_{1,1}\right) .
$$

Since $\beta_{*} L_{i, 1}=\zeta^{5} L_{i, 1}$, there exist constants $\lambda_{0}, \ldots, \lambda_{3} \in \mathbf{C}$ such that P.D. $\left(\omega_{1,1}\right)=$ $\sum_{i=0}^{3} \lambda_{i} L_{i, 1}$. The result follows from Proposition 3.4 and the equations

$$
\begin{aligned}
& \int_{\gamma_{0,0}} \omega_{1,1}=\left(\text { P.D. }\left(\omega_{1,1}\right), \gamma_{0,0}\right)=\sum_{i=0}^{3} \lambda_{i}\left(L_{i, 1}, \gamma_{0,0}\right)=\left(\zeta^{5}-\zeta\right) \lambda_{0}+(\zeta-1) \lambda_{1}, \\
& \left.\int_{\gamma_{1,0}} \omega_{1,1}=\text { P.D. }\left(\omega_{1,1}\right), \gamma_{1,0}\right)=\sum_{i=0}^{3} \lambda_{i}\left(L_{i, 1}, \gamma_{1,0}\right)=(1-\zeta) \lambda_{0}+\left(\zeta^{5}-\zeta\right) \lambda_{1}+(\zeta-1) \lambda_{2}, \\
& \left.\int_{\gamma_{2,0}} \omega_{1,1}=\text { P.D. }\left(\omega_{1,1}\right), \gamma_{2,0}\right)=\sum_{i=0}^{3} \lambda_{i}\left(L_{i, 1}, \gamma_{2,0}\right)=(1-\zeta) \lambda_{1}+\left(\zeta^{5}-\zeta\right) \lambda_{2}+(\zeta-1) \lambda_{3}, \\
& \left.\int_{\gamma_{3,0}} \omega_{1,1}=\text { (P.D. }\left(\omega_{1,1}\right), \gamma_{3,0}\right)=\sum_{i=0}^{3} \lambda_{i}\left(L_{i, 1}, \gamma_{3,0}\right)=(1-\zeta) \lambda_{2}+\left(\zeta^{5}-\zeta\right) \lambda_{3} .
\end{aligned}
$$


A nontrivial algebraic cycle in the Jacobian variety of the Fermat sextic 37

Let $\int_{L_{i, k}} \omega_{r, s} \omega_{l, m}$ denote $\sum_{n=0}^{5} \zeta^{n k} \int_{\gamma_{i, n}} \omega_{r, s} \omega_{l, m}$.

Lemma 4.2. For $i=0, \ldots, 3$, we have

$$
\int_{L_{i, 1}} \omega_{1,2} \omega_{1,3}=6\left\{\zeta^{2 i}(1+\zeta)\left(x_{1,2,1,3}-1\right)-\zeta^{i}\right\}
$$

Proof. By Theorem 3.6, it is easy to compute

$$
\begin{aligned}
\int_{\gamma_{i, n}} \omega_{1,2} \omega_{1,3}= & \zeta^{2 i-n}(1+\zeta)\left(x_{1,2,1,3}-1\right)+2\left(1-\zeta^{2 n}\right) \zeta^{i+3 n}(1-\zeta) \\
& -\left(1-\zeta^{3 n}\right) \zeta^{i+2 n}(1-2 \zeta)
\end{aligned}
$$

Using this equation, we obtain the result in a straightforward way.

TheOREM 4.3. Let $F(6)$ be the Fermat sextic. Then, the cycle $F(6)-F(6)^{-}$is not algebraically equivalent to zero in $J(F(6))$.

Proof. By the definition of the harmonic volume $I_{R}$, we have

$$
I_{R}\left(\omega_{1,2} \otimes \omega_{1,3} \otimes \omega_{1,1}\right) \equiv \sum_{i=0}^{3} \lambda_{i} \int_{L_{i, 1}} \omega_{1,2} \omega_{1,3} \bmod R
$$

Using Lemma 4.1 and 4.2, we obtain

$$
2 I_{R}\left(\omega_{1,2} \otimes \omega_{1,3} \otimes \omega_{1,1}\right) \equiv \frac{6}{61}\left\{(42-3 \zeta) x_{1,2,1,3}-95+46 \zeta\right\} \bmod R
$$

and denote it by $\alpha$. By Lemma 3.8 and the numerical calculation (Figure 1 in Appendix), we obtain the value

$$
2 \Re(\alpha) \equiv \frac{6}{61}\left(81 x_{1,2,1,3}-144\right) \equiv 0.74286 \pm 1 \times 10^{-5} \bmod \mathbf{Z}
$$

The result follows from Theorem 2.2 and the lemma

$$
2 \Re(\alpha) \notin \mathbf{Z} \Rightarrow \alpha \notin \mathbf{Z}[\zeta]
$$




\section{Appendix}

We introduce the Mathematica program [11] in the proof of Theorem 4.3.

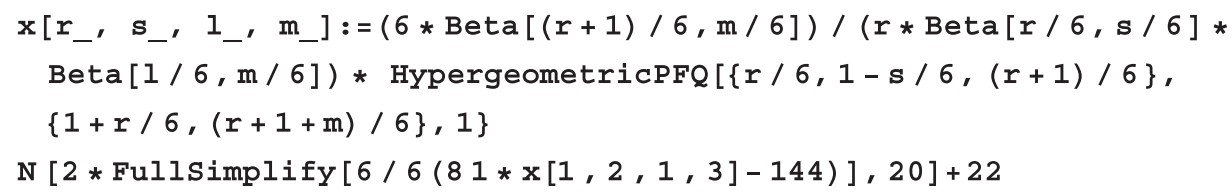

Figure 1. A numerical calculation program in the proof of Theorem 4.3

\section{References}

[1] Ceresa, G.: $C$ is not algebraically equivalent to $C^{-}$in its Jacobian. Ann. of Math. (2) 117 (1983), no. 2, 285-291.

[2] Chen, Kuo Tsai: Algebras of iterated path integrals and fundamental groups. Trans. Amer. Math. Soc. 156 (1971), 359-379.

[ 3 ] Gross, Benedict H.: On the periods of abelian integrals and a formula of Chowla and Selberg. With an appendix by David E. Rohrlich. Invent. Math. 45 (1978), no. 2, 193-211.

[4] Gunning, R. C.: Quadratic periods of hyperelliptic abelian integrals. Problems in analysis (Papers dedicated to Salomon Bochner, 1969), pp. 239-247. Princeton Univ. Press, Princeton, N.J., 1970.

[5] Harris, Bruno: Harmonic volumes. Acta Math. 150 (1983), no. 1-2, 91-123.

[6] Harris, Bruno: Homological versus algebraic equivalence in a Jacobian. Proc. Nat. Acad. Sci. U.S.A. 80 (1983), no. 4 i., 1157-1158.

[ 7 ] Harris, Bruno: Iterated integrals and cycles on algebraic manifolds. Nankai Tracts in Mathematics, 7. World Scientific Publishing Co., Inc., River Edge, NJ, 2004.

[8] Kamata, Yasuo: The algorithm to calculate the period matrix of the curve $x^{m}+y^{n}=1$. Tsukuba J. Math. 26 (2002), no. 1, 15-37.

[9] Tadokoro, Yuuki: A nontrivial algebraic cycle in the Jacobian variety of the Klein quartic. Math. Z. 260 (2008), no. 2, 265-275.

[10] Tretkoff, C. L.; Tretkoff, M. D.: Combinatorial group theory, Riemann surfaces and differential equations. Contributions to group theory, 467-519, Contemp. Math., 33, Amer. Math. Soc., Providence, RI, 1984.

[11] Wolfram, Stephen: The Mathematica book, Fourth edition. Wolfram Media/Cambridge University Press, 1999.

Natural Science Education

Kisarazu National College of Technology

2-11-1 Kiyomidai-Higashi, Kisarazu

Chiba 292-0041, Japan

E-mail address: tado@nebula.n.kisarazu.ac.jp 